\title{
LAS ABUELAS COMO CUIDADORAS: UNA VISIÓN ECOLÓGICA DE SU ROL
}

\author{
Melisa Judith Ortiz Barrero ${ }^{62}$
}

\section{RESUMEN}

El presente artículo de reflexión teórica tiene como objetivo explorar los aspectos involucrados en el hecho de convertirse en abuelo o abuela, su función de cuidadora familiar y sus redes de apoyo (relaciones afectivas o laborales) desde una perspectiva ecológica. En Colombia, las familias valoran el rol de abuela cuidadora, particularmente en la conformación de familias extendidas, pues se convierte en un recurso social para las personas (en cuanto al cuidado de los niños) y una fuente de apoyo emocional en situaciones difíciles (separación, divorcio, maternidad adolescente, viudez, desplazamiento forzado). Además, de que se favorece un mejor aprovechamiento de los recursos económicos, por un trabajo no remunerado. En cuanto al rol de la abuela como cuidadora y las ya mencionadas redes, en las cuales está involucrada o puede llegar a acceder, se destaca una posible sobrecarga en sus labores y el estrés debido al cuidado que brindan como red de apoyo familiar, aunque este hecho también puede estar relacionado con aislamiento o falta de otras redes de apoyo que cuiden de ella. Finalmente, se presentan algunas posibilidades de investigación e intervención para identificar y favorecer las redes de apoyo de las abuelas.

Palabras clave: redes sociales, redes de apoyo, familia extensa, rol de abuelo, abuelas cuidadoras.

\begin{abstract}
This theoretical article aims to explore the issues involved in the fact of becoming grandparent, the role of caregiver and family support networks from an ecological perspective.

In Colombia families value the role of caregiver for the grandmother, particularly in the formation of extended families is a social resource for people (in terms of child care) and a source of emotional support in difficult situations (separation, divorce, teenage motherhood, widowhood, forced displacement), in addition to fostering better exploitation of economic resources.
\end{abstract}

Regarding the role of grandmothers as caregivers and support networks which is involved in or could access highlights a possible overload in their work and stress due to the care they provide as a network of family support, but this also may be related to isolation or lack of other support networks to care for her. Finally presents some opportunities for research and intervention to identify and encourage the support networks of grandmothers.

62 Pregrado en Psicología. Maestría en Psicología, Universidad de los Andes. Docente tiempo completo de la Universidad Nacional Abierta y a Distancia, UNAD en el CEAD José Acevedo y Gómez, pertenece al grupo de investigación que apoya la maestría de psicología de la UNAD. E-mail: melissa.ortiz@unad.edu.co 
Key words: social networks, support networks, extended family, role of grandfather, grandmother caregivers.

Recibido: 1 de mayo de 2009

Aceptado: 3 de agosto de 2009

\section{INTRODUCCIÓN}

En países latinoamericanos las abuelas ocupan un papel valioso dentro de la familia y se involucran de manera significativa en la vida de sus nietos; con frecuencia se encargan del cuidado parcial o total de sus nietos; en muchos casos viven con ellos y son las responsables de suplir las necesidades elementales del niño. A pesar de la importancia que tienen en el rol que desempeñan como red de apoyo familiar y social, en la psicología, este rol no ha recibido la importancia que merece y, particularmente en Colombia, los estudios sobre esta población son muy limitados.

Dentro de este contexto, al hablar de redes se destaca que son una estrategia importante en la estructuración de la calidad de vida de toda persona a nivel individual y familiar. Las redes socio-familiares se caracterizan por la solidaridad y la expresión de afecto, y se convierten en una fuente de protección y apoyo, también, en medio de crisis ecológicas (en sus diferentes niveles de relación).

Es por esto que en el presente artículo de reflexión teórica se busca explorar los aspectos psicosociales involucrados en el hecho de convertirse en abuelo o abuela en Colombia, las características de la etapa del desarrollo en el que se encuentran los abuelos, las implicaciones de este rol y la función que estos pueden desempeñar dentro de la familia de acuerdo con el contexto social, sus propias redes de apoyo, así como presentar datos ilustrativos sobre los sistemas familiares en Colombia, particularmente la familia extensa y, desde una perspectiva ecológica, enfatizar en el rol de las abuelas como cuidadoras.

Finalmente, se busca plantear algunas perspectivas de investigación e intervención en abuelas cuidadoras para realizar contribuciones a este campo poco explorado.

\section{Envejecimiento y aspectos psicosociales involucrados}

En Colombia la esperanza de vida de los hombres y las mujeres se ha incrementado significativamente en las últimas décadas. De los 50 años de vida en promedio hacia 1950, en la actualidad la expectativa de vida ha aumentado a los 70 años. Pero específicamente la longevidad femenina es mayor. Actualmente una mujer de 60 años puede llegar a vivir más de 20 años adicionales y un hombre de 5 a 10 años adicionales. Así, en la población mayor de 60 años el número de mujeres excede en 23\% al de los hombres (DANE, 2000; DANE, 2003).

El concepto de envejecimiento comprende desde la adultez media (muchos de los problemas del adulto mayor tienen origen en la adultez media y se les da continuidad en la adultez tardía) 
hasta la vejez. En la adaptación positiva de este proceso tienen gran influencia las acciones y actitudes de la sociedad en la que la persona envejece. La vejez no es una enfermedad, es un proceso multifacético de maduración; se evidencia un declive en la energía física, se es más susceptible a enfermedades y comienza a hacerse más difícil la integración personal y la orientación en la sociedad.

En la sociedad actual, la calidad de vida se asocia con tener una capacidad adquisitiva para vivir con todas las necesidades básicas cubiertas, disfrutar de salud psíquica, física y relaciones sociales satisfactorias. Mundialmente el número de personas que se encuentra en la vejez ha aumentado, la sociedad se enfrenta a nuevos retos y da fuerza al reciente concepto de solidaridad entre las generaciones y entre distintos grupos debido a los continuos cambios sociales, a las crisis y a la inseguridad en los sistemas sociales. Un rol característico de la adultez tardía es el de abuelo(a), es así que en cierta medida el nieto viene a compensar el vacío que dejó el hijo al salir del hogar. Una de las funciones que se cumple en este rol es la de dar continuidad y transmitir tradiciones familiares.

La calidad de vida en la vejez tiene que ver con la inclusión social que debe ser garantizada por el gobierno en cuanto a seguridad económica e infraestructuras de apoyo, pero también tiene que ver con la conformación de redes sociales. Algunos autores han estudiado diferentes variables que influyen en la calidad de vida. Granzin y Haggard (2000) sostienen que el comportamiento humano está básicamente influenciado por la interacción entre el organismo y el ambiente, es decir, la persona elige comportarse de cierta manera en relación con el contexto correspondiente. El modelo interaccionista de estos autores comprende tres aspectos: la persona, que hace referencia a edad, educación, ingresos y funcionamiento físico; el ambiente, como ej. tener casa propia, apoyo emocional e identidad con la comunidad y las experiencias subjetivas como sentir malestar por estar desempleado y la percepción de salud mental y de satisfacción con la vida. Por ejemplo, la satisfacción con el trabajo puede relacionarse con el grado de adaptación entre la persona y el ambiente.

Otras aproximaciones sugieren que las personas participan en tareas del desarrollo que tienen un valor personal y cultural las cuales cambian a lo largo de la vida (de acuerdo con factores del contexto); en la realización de estas tareas los rasgos de personalidad (como extraversión) y los recursos tangibles (como altos ingresos económicos) tienen efectos directos sobre el bienestar. Así, éste puede incrementarse cuando los individuos son capaces de seguir sus distintas metas personales de forma realista en su vida diaria; cuando las personas participan en las actividades que desean y se adecuan a sus características y necesidades personales experimentan mayor bienestar; es decir, cuando hacen una evaluación realista de sí mismas y de las oportunidades que ofrece el contexto al que pertenecen.

Además, las actividades más apreciadas por las personas pueden mantenerse o modificarse en el tiempo sin necesidad de que deje de experimentarse bienestar (Cantor \& Sanderson, 1999, Liberalesso, 2002). Por ejemplo, si una persona tiene la oportunidad de realizar las tareas del desarrollo propias de su etapa, puede sentirse más satisfecha (como en el caso de ser abuelo a finales de la adultez media) en comparación con otra que deja de realizar alguna 
tarea o que se ve obligada a realizarla a destiempo (como en el caso de ser abuela en la adultez temprana o antes de los 45 años); es por eso que cumplir con una tarea en el tiempo adecuado genera mayor satisfacción.

Por otra parte, el apoyo de las amistades y las relaciones familiares contribuye a una mejor calidad de vida física y emocional de las personas; lo cual está asociado con la necesidad de pertenencia y de crear vínculos con otros, presente en los individuos desde el momento del nacimiento (Myers, 1999).

Myers (1999) sugiere que este sentido de pertenencia varía dependiendo del tipo de cultura en la que se desarrolle el individuo. Las culturas occidentales típicamente valoran el individualismo, pero las culturas de Asia y del tercer mundo (entre las que se encuentra la Latinoamericana) dan gran valor al colectivismo; las personas dan más prioridad a las metas y al bienestar de sus familias y de los grupos a los que pertenecen. En dichas culturas colectivistas, es apreciada la solidaridad y las redes sociales contribuyen a la definición del auto-concepto y proveen una identidad social; tal es el caso de las familias extensas en las cuales sus miembros asumen responsabilidades mutuas y se crea una auto-confianza e identidad grupal.

De acuerdo con esto, se puede decir que para los latinoamericanos, las relaciones familiares son muy importantes en la vida y, por lo tanto, el bienestar personal está relacionado con el bienestar familiar. En general, en culturas como ésta las personas sienten la necesidad de ayudar a un familiar cuando éste lo necesita y en caso de necesitar ayuda acuden, en primer lugar, a un familiar cercano tal es el caso de las madres adolescentes o madres trabajadoras que buscan la ayuda de sus propias madres para el cuidado de sus hijos.

\section{Familia extendida y el rol de los abuelos(as)}

Dentro de las familias que residen en la misma vivienda en Colombia, prevalece la familia nuclear, que para 1993 reunía el 54.9\% de las familias, seguida de la familia extendida (o extensa), que reunía el 30.4\%. Así, predomina la modalidad de la pareja con hijos que no comparte el espacio con ninguna otra persona y en segundo lugar, está la de pareja con hijos que vive con otros parientes (DANE, 1998). Esta considerable proporción de familias extendidas está relacionada en parte con el género del jefe de hogar, que es un factor determinante en la organización del núcleo familiar. Cuando el hombre es la cabeza del hogar o cuando la pareja comparte dicho rol, con frecuencia organizan su núcleo familiar contando solamente con la presencia de sus hijos (en un espacio independiente). En caso contrario, cuando las mujeres son cabezas de familia aunque en su mayoría conviven sólo con sus hijos (35\%), una cuarta parte de ellas (26.4\%) conviven con sus hijos y otros parientes (generalmente sus padres); por lo tanto, la mujer jefe parece requerir más del apoyo de otros parientes para poder sobrevivir (DANE, 2005).

La familia entendida como grupo es la estructura social básica: "se entiende por grupo el conjunto de personas que participan en una misma estructura vivencial colectiva.... No 
es sólo la suma de individuos... debe tener una finalidad compartida y los miembros estar comunicados entre sí” (Santana, 2003, p.119).

Comúnmente, la familia extendida se conforma cuando el progenitor(a) sólo(a) con sus hijos reside con sus padres o hermanos, inicialmente para poder asumir la supervivencia de los hijos pequeños y posteriormente para cuidar de los padres ancianos. La cantidad de familias extendidas aumenta con la ruptura de las uniones (separación, divorcio), con el proceso de envejecimiento y la viudez de los progenitores (DANE, 1998).

En concordancia con lo anterior en cuanto a la familia, Dychtwald (1995, citado por DulceyRuiz \& Uribe, 2002) expone que la familia nuclear (padres e hijos) en el transcurrir de las últimas décadas ha pasado a ser la familia multigeneracional (grupos conformados por personas de varias familias y de más de dos generaciones). De acuerdo con Dulcey-Ruiz \& Uribe (2002) las familias de tres generaciones serán las más representativas en el año 2020; así, los requerimientos de cuidado por parte de los miembros más pequeños y de los ancianos serán una fuente de estrés para los miembros que se encuentren en su adultez media.

La familia extensa cumple una serie de funciones para los miembros que la conforman: por una parte sirve de apoyo para el buen desarrollo físico y psicológico de algunos familiares dependientes, particularmente los que se encuentran en la infancia y la niñez; por otra parte, la red de relaciones que se establece en la familia extendida (por ejemplo, cuando se convive con los abuelos) puede actuar como un factor de protección frente a problemas familiares y sociales, dando la oportunidad de brindar y recibir ayuda económica para suplir las necesidades básicas de los miembros y de proveer apoyo emocional en situaciones críticas como cuando se es madre soltera, separada, divorciada, viuda o se es madre adolescente.

A pesar de que las abuelas siempre han tenido una función de apoyo familiar y social, el rol de abuela cuidadora es un fenómeno poco estudiado; pero el aumento de la esperanza de vida de la mujer y el hecho de que esta participe del mundo laboral, ha producido un cambio en sus roles tradicionales lo cual ha abierto un nuevo campo de investigación. Las abuelas continúan deseando ser un apoyo para su familia, pero en la actualidad son mas autónomas, tienen una vida social más amplia y desean desarrollar sus propias vidas aún en edad avanzada, por lo tanto, ser o no cuidadoras de sus nietos se convierte en un dilema conflictivo que les impone una transición de roles (Villalba, 2002).

Existen datos que indican que la relación abuela-nieto es más importante de lo que comúnmente se cree y llega a ser la segunda más importante después de la relación con los padres (Brussoni \& Boom, 1998, Sanders \& Trygstad, 1993 citados por Harwood, 2002; Kornhaber, 1996; Westheimer \& Kaplan, 2000). Se ha encontrado que en la relación de los abuelos con sus nietos, ellos suelen expresar y sentir amor hacia el nieto, estar orgullosos de sus características y logros y trasmitirles conocimientos (Harwood, 2002).

En la actualidad, el ser abuelo se ha convertido en uno de los roles que desempeñan las personas en la etapa de la adultez. Particularmente en familias multigeneracionales, las 
personas que se convierten en abuelos durante la adultez media siguen desempeñando gran parte de sus funciones como padres y además su rol de abuelos lo cual puede generar mayores tensiones familiares. En Colombia, como en otros países, se considera que los abuelos son de gran importancia y están siempre dispuestos a brindar ayuda a sus hijos y nietos; es así que cuando las abuelas cuidan de sus nietos de manera permanente o frecuente, pueden cumplir una función más trascendental y ser de gran ayuda para el desarrollo integral del nieto. Ser abuela es un evento significativo e implica gran cantidad de tiempo en la vida, por lo tanto, se le debe prestar mayor atención

$\mathrm{Al}$ evaluar las relaciones intergeneracionales entre abuelos y nietos, desde el punto de vista de los abuelos, se observa un alto grado de satisfacción por parte de los abuelos en la relación que mantienen con sus nietos. El enriquecimiento personal de la abuela en su rol con el nieto/a proviene tanto de la relación con su nieto como del cuidado que ella le puede brindar (Ortiz y Carrillo, 2007). La tarea de cuidar al nieto es algo importante para ellas ya que disfrutan al sentirse capaces, responsables, útiles y necesarias. Además, este es un rol reconocido y valorado a nivel familiar, (Benlloch, Llopis, Bejarano \& Pinazo, 1996).

Según Craig (2001) los roles que pueden desempeñar los abuelos y las abuelas básicamente son: como compañía (estar allí en caso de ser necesitados), como guardián de la familia (ante una emergencia familiar), como árbitro (mediar en los conflictos entre hijos y nietos) y como aquel que conserva la memoria histórica de la familia (transmitiendo tradiciones). La mayoría de personas mayores de 65 años consideran que sus nietos son muy importantes y reportan verlos por lo menos una vez a la semana. Este autor plantea que a medida que los abuelos aumentan en edad, su papel puede ser más limitado; a pesar de esto, los vínculos emocionales entre ellos y sus nietos persisten y tienen un impacto significativo en el desarrollo de los niños.

Belsky (1996) sugiere que para la mayoría de personas de edad, ser abuelo tiene un significado vital, porque en este papel se es valioso e importante no por los logros o por lo que se hace, sino por el hecho de "estar allí". En momentos de crisis los abuelos se convierten en estabilizadores de la situación familiar, pueden ayudar a sus nietos indirectamente (orientando en pautas de crianza y en la educación de los niños), pueden actuar como mediadores (ayudando a resolver problemas entre los niños o adolescentes y sus padres), y contribuyen a mantener la unidad familiar (como punto de encuentro en reuniones familiares) (Belsky, 1996; Ochaita \& Espinoza, 1995).

La influencia de los abuelos en el desarrollo de los niños varía dependiendo del estilo de interacción entre ellos y sus nietos (Ortiz y Carrillo, 2007). Según Barber (2001) existen cinco estilos de ser abuelos. El estilo formal: a este estilo pertenecen aquellos abuelos que se preocupan por no interferir en la crianza de sus nietos, pero que se mantienen muy interesados en el bienestar de estos y se comportan de una manera complaciente con los niños (por lo general, a este grupo pertenecen los abuelos más ancianos); el estilo divertido: generalmente estos abuelos tienen una actitud alegre, juegan y disfrutan su tiempo libre con los nietos (con frecuencia están en la adultez media); al estilo distante de abuelos corresponden los que son complacientes pero alejados, que sólo tienen contacto con sus nietos en días especiales; 
el estilo de abuelo llamado padre sustituto corresponde a aquellos que asumen el rol de cuidador principal del nieto cuando los padres no pueden o están ausentes para cuidarlo (esto se observa principalmente en las abuelas); por último, el estilo depósito de sabiduría, al cual pertenecen los abuelos que constantemente brindan herramientas y consejos al nieto (principalmente los abuelos hombres).

Aunque esta clasificación ilustra algunos estilos de interacción de los abuelos, es al mismo tiempo limitada; el estilo y comportamiento de un abuelo puede cambiar de un nieto a otro o de un momento y contexto especifico a otro. Adicionalmente, el comportamiento de un abuelo puede corresponder a varios estilos.

\section{Las abuelas en Colombia}

Colombia es uno de los países donde los abuelos son de gran importancia y parte fundamental de la red de apoyo de los hijos y nietos. El rol de abuelo está particularmente colmado de emociones, se puede expresar libremente el afecto y no representa una carga de responsabilidades; excepto cuando se está involucrado en el cuidado del nieto, cuando se es abuelo prematuramente (por ser padre de una madre adolescente) o cuando se vive en un hogar multigeneracional, lo cual puede disminuir la satisfacción. Ser abuelo parece ser gratificante para la mayoría de las personas, pues les permite sentirse queridas, reconocidas y necesarias para el nieto (Ortiz y Carrillo, 2007).

La psicología se ha interesado cada vez más por el papel que cumplen los abuelos en la familia, lo cual se debe en parte al incremento en la tasa de divorcios en las últimas décadas, ya que los abuelos, como parte fundamental de la red de apoyo de los hijos, entran a ocupar un rol más importante en la vida de sus nietos en caso de que los padres se divorcien. Este fenómeno ha permitido observar la importancia de los abuelos en la crianza y el desarrollo de los niños; cuando el bienestar del niño y la mayoría de responsabilidades le corresponden predominantemente a uno de los padres del niño (comúnmente la madre), los abuelos pueden brindar una ayuda considerable. Los estudios actuales sobre abuelos ya no se limitan a reportar la cantidad de contactos de los abuelos con sus hijos y nietos sino que analizan las características de esta relación y las variables que influyen en la misma (Rosenthal, 2000; Westheimer \& Kaplan, 2000).

En Colombia este interés está surgiendo en gran parte por las condiciones socioeconómicas del país (Gamboa, 2007), por el aumento de embarazos en adolescentes y de separaciones familiares que obligan a que en una misma vivienda residan tres generaciones y que llevan a los abuelos a ejercer una influencia determinante en el desarrollo de la familia y de los niños. Estas circunstancias contribuyen a que la unión familiar se convierta en una alternativa para suplir necesidades físicas y emocionales.

Los colombianos en este nuevo siglo tienen la oportunidad no sólo de vivir más años, sino de vivir muchas más experiencias y compartir más tiempo con su familia (hijos y nietos); 
además, dada la alta proporción de mujeres que tienen hijos antes de los 25 años, las abuelas tienen la oportunidad de interactuar con sus nietos y bisnietos.

Teniendo en cuenta los datos nacionales sobre una mayor expectativa de vida en las mujeres y su situación como jefes del hogar, es posible pensar que en las familias extensas hay una mayor presencia de abuelas y una mayor influencia de ellas en la vida de sus hijos y nietos. Las abuelas pueden asumir roles parentales sustitutos al brindar cuidado de manera ocasional o permanente a sus nietos (Carrillo y cols., 2004).

Además, el hecho de pertenecer a una familia extendida y convivir con el nieto ha implicado una mayor participación en la crianza y cuidado de este; pero también en los casos en que los padres se ausentan por largos períodos de tiempo durante el día debido a su trabajo, la abuela suele participar activamente en la vida del nieto así no convivan juntos.

Las abuelas que habitualmente realizan actividades con los nietos y participan en su cuidado, ayudan a su crianza, a su proceso de socialización (permitiéndoles crear vínculos afectivos y sirviendo de modelos de relación) y al desarrollo de habilidades cognitivas y motrices. Ainsworth (1989) observó que en edades tempranas todos los bebés que llegan a estar apegados a sus madres también llegan a estar apegados a alguna otra figura familiar como el padre, la abuela, u otro adulto de la casa, o a un hermano mayor.

De acuerdo con Carrillo \& cols. (2004) los abuelos, particularmente, desempeñan un rol importante en la vida familiar y en el desarrollo social de los niños; ellos son una fuente de apoyo social, emocional y afectivo, de hecho se pueden llegar a considerar la red de apoyo social más cercana para los padres. En el caso de las madres adolescentes estas suelen convivir en un sistema familiar de tres generaciones conformado por abuelos (puede ser sólo la abuela), madre adolescente y bebé. En este sistema, la madre adolescente y su hijo reciben apoyo social, emocional y financiero de sus padres y la abuela muestra una mayor influencia en el desarrollo del nieto.

Son escasos los estudios realizados con abuelas colombianas pero en los consultados para esta investigación se evidencia la importancia de los sistemas familiares de tres generaciones y el fenómeno de la familia extendida donde las abuelas ejercen mayor influencia en los nietos y en otros miembros de la familia. En el rol de abuela cuidadora, se destacan los sentimientos y emociones positivos que se dan en la relación con el nieto y el fuerte vínculo que se crea (especialmente con la abuela materna) (Carrillo y Maldonado, 2003; Ortiz y Carrillo, 2007). Sin embargo, estos resultados no reflejan la existencia de sistemas de apoyo para las abuelas como cuidadoras familiares, las cuales pueden llegar a considerarse un grupo de la población con necesidades particulares y en posible situación de riesgo psicosocial.

\section{Abuelas como cuidadoras familiares y redes de apoyo}

Definiendo apoyo emocional como una red de relaciones sociales que actúan a modo de mecanismo protector en una situación de difícil afrontamiento o que son consideradas para 
el individuo como estresantes, algunas investigaciones latinoamericanas han mostrado que la ausencia de apoyo social en madres (o cuidadoras) que desempeñan múltiples roles, es una fuente generadora de estrés, el cual puede influir negativamente en la salud física y mental, el bienestar y la productividad (Gómez,2003; Meneses, Feldman \& Chacón; 1999).

Las relaciones cercanas influyen en el nivel de salud de los individuos; es frecuente encontrar que estas relaciones contribuyen a la salud y a la felicidad, pues quienes tienen más relaciones cercanas con amigos, con la familia, con miembros de la iglesia, compañeros de trabajo o miembros de otros grupos de apoyo, tienen menores posibilidades de morir prematuramente; además las personas con fuertes vínculos sociales se alimentan mejor y se ejercitan más (Myers, 1998; Gamboa y Cordero, 1998).

Adicionalmente, el apoyo emocional y el establecimiento de redes sociales significativas son cruciales en el afrontamiento de la crianza de los niños y en las expectativas concernientes a la paternidad, pues pueden tener un efecto muy positivo en el bienestar psicológico de los cuidadores. Brooks-Gunn y Chase-Lansdale (1995) identificaron dos efectos positivos cuando las madres adolescentes corresiden con las abuelas cuidadoras: (a) las abuelas pueden proveer apoyo económico y emocional cuando las madres adolescentes habitan con las abuelas y (b) cuando los recursos son escasos, es el apoyo mutuo tanto económico como emocional entre madres y abuelas, el factor que puede intervenir de forma efectiva en la crianza y en el estrés del rol de cada una.

Destacando el papel prioritario que desempeñan los abuelos dentro de las relaciones familiares, Rubio y Garrido (1995) investigaron el grado de actividad social entre abuelos y nietos, además de otros aspectos intrínsecos de las redes sociales estimados en dos dimensiones: el tiempo de relación entre ambas generaciones y el cariño depositado o el gusto por la compañía de los mayores. Así, encontraron que los abuelos y los nietos mantienen un contacto muy frecuente y el cariño depositado mutuamente es elevado.

En cuanto a las actitudes, los sentimientos y las emociones que interactúan en la definición individual del rol de abuela y cuál es el significado que tiene para ella el cuidado que brinda a sus nietos Budini y Mussatti (1998) llevaron a cabo un estudio con abuelas en el sugieren que el cuidado de los niños por parte de sus abuelos trae varios beneficios; la interacción abuelanieto se convierte en una oportunidad para ofrecer ayuda material o económica, contribuir a las relaciones interpersonales de la abuela y para compartir experiencias entre diferentes generaciones. Particularmente, el cuidado de la abuela al niño menor de tres años es muy importante cuando la madre trabaja, siendo así aquella, el cuidador principal o el suplemento de otras formas de cuidado extra-familiar. Estos investigadores también encontraron que la disponibilidad de la abuela y el hecho de involucrarse en el cuidado de sus nietos, están determinados no solo por las necesidades materiales de la madre, sino fundamentalmente por los patrones de crianza que ella experimentó durante su crecimiento; es decir, que cuando las abuelas habían observado en su familia de origen este tipo de relación de ayuda entre sus padres y abuelos, era más fácil que accedieran a cuidar de sus nietos sin manifestar mayores conflictos. 
Por su parte, algunos autores han analizado los roles familiares particularmente el rol de cuidador a la luz de la perspectiva ecológica del desarrollo humano propuesta por Bronfenbrenner \& Ceci (1994), la cual ha aportado importantes bases teóricas de investigación para la psicología comunitaria. Dentro de esta perspectiva los roles, estilos y condiciones de vida se desarrollan de acuerdo con los espacios y modalidades de la actividad humana y formas de interacción, correspondientes a los diferentes sistemas que influyen y que componen el ambiente ecológico. Estos sistemas son: microsistema (contextos cercanos de interacción como la familia y la escuela), mesosistema (familia, barrio y redes sociales), exosistema (interacción entre los microsistemas), macrosistema (influencia de creencias y aspectos socioculturales, políticos y económicos) y cronosistema (hechos históricos y biográficos). Dulcey-Ruiz y Uribe (2002) y Villalba (2002) enfatizan la importancia de esta perspectiva ecológica y cultural en el estudio de los cuidadores familiares. Bajo este marco teórico, el rol de cuidador y la percepción que los adultos tengan de dicho rol está altamente influenciado por diferentes factores personales, comunitarios y sociales propios del contexto en el cual ellos se desenvuelven (ver Figura 1).

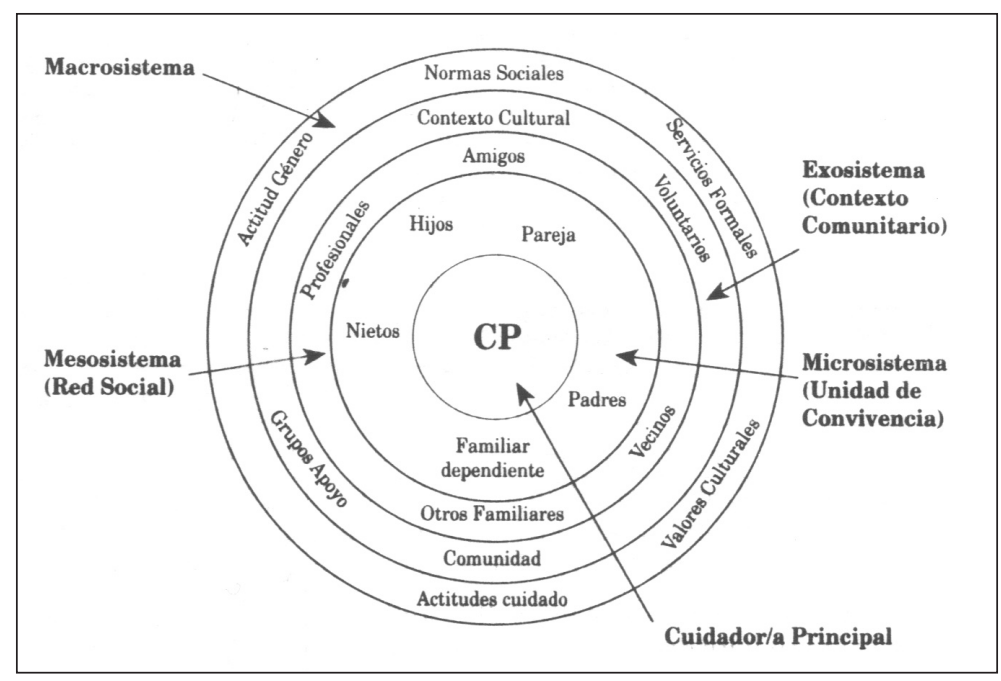

Figura 1. Representación ecosistémica de los cuidados familiares (tomado de Villalba, 2002a)

En este campo de investigación reciente sobre los cuidados y cuidadores familiares, la perspectiva ecológica ha permitido analizar más allá de las causas inmediatas que generan la situación de cuidado, ha abarcado influencias históricas complejas que tienen injerencia en los cuidados familiares, como las transiciones de rol, las tradiciones, los parámetros sociales y la cultura. Este enfoque integra la cultura como un concepto transversal, particularmente para las investigaciones sobre cuidados familiares la cultura se considera como contexto (teoría de sistemas ecológicos) (Cooper y Denner, 1998). Así, en el futuro de la intervención comunitaria cada vez es más necesario tener en cuenta la dimensión cultural y contextual para formar conceptos y realizar acciones. Es importante tener presente que "las características de personalidad de los cuidadores, sus experiencias, sistemas de creencias y costumbres 
familiares están determinadas en gran parte por factores históricos y culturales que se han de tener presentes" (Villalba, 2002, p. 47).

Dentro de esta perspectiva, es importante conocer las transformaciones ecológicas para entender las implicaciones que estas tienen en los cuidados familiares; es decir, conocer los cambios de roles que se dan cuando un miembro de la familia se convierte en cuidador principal o secundario de un familiar dependiente; frecuentemente, a partir de situaciones estresantes o críticas de la vida (como es el caso de las abuelas en familias con madres adolescentes). Así, "la capacidad de respuesta y de adaptación de las personas (recursos internos) y apoyo social con el que cuente (recursos externos) serán determinantes a la hora de valorar los costos y recompensas de dichas transiciones ecológicas" (Villalba, 2002a, p. 49). Las mujeres culturalmente han asumido más que los hombres el rol de cuidador familiar; aunque últimamente ha aumentado la participación de ellos en las tareas de cuidado dentro de la familia, dicha participación continúa siendo mayor en las mujeres.

Las redes de apoyo de las abuelas cuidadoras están conformadas principalmente por sus familiares, pero también por amigos y otras personas más o menos cercanas las cuales, en determinado momento, se pueden integrar a los cuidados como círculo de apoyo (Villalba, 2002b). El estudio de las redes de apoyo de los cuidadores se hace necesario en el campo de las abuelas cuidadoras, ya que esto puede influir en la calidad de los cuidados brindados y en el bienestar físico y psicológico de las abuelas. La calidad de la relación y el dar y recibir apoyo depende de la interacción de determinantes contextuales como: la personalidad y la autoestima (nivel intrapersonal), el tipo de relación y los conflictos (nivel interpersonal) y los eventos vitales y el nivel socioeconómico (nivel situacional).

El apoyo social, como ya se ha mencionado, está relacionado con los cuidados familiares y la ayuda mutua entre miembros de la familia. Cuando los cuidados son prolongados se puede presentar el estrés y afectar los sistemas de apoyo de los cuidadores. El concepto de apoyo social es de gran aplicación en la psicología social, se define como aquellas relaciones sociales que hacen sentir valiosas y queridas a las personas y los hacen percibir que otros están disponibles a ofrecerles asistencia y apoyo si lo necesitan. Así, en los cuidados familiares este apoyo no solamente contribuye al bienestar del familiar dependiente sino también de los cuidadores. Se deben dar permanentemente procesos de intercambio de ayuda (material, afectiva e instrumental). Lo que determina si los cuidados familiares proveen o no apoyo social son los significados que atribuyan a su interacción los cuidadores y las personas que están siendo cuidadas (Martínez \& García, 1995).

De acuerdo con lo anterior se pueden plantear tres sistemas de ayuda que pueden interactuar en el caso de las abuelas cuidadoras y otros cuidadores familiares: el natural (incluye familia, vecinos y amigos); el natural organizado o informal (asociaciones y grupos de apoyo) y el formal o profesional (servicios profesionales públicos o privados). Todos estos sistemas deben articular estrategias y coordinar acciones, en especial el sistema formal de apoyo se debe afirmar para fortalecer los sistemas naturales (Santana, 2003). Por lo tanto, en las familias con presencia de abuelas cuidadoras se pueden desarrollar programas de 
intervención con grupos y con la comunidad; con grupos principalmente para favorecer las redes de relaciones y potenciar habilidades; y con la comunidad para sensibilizar a la población sobre las problemáticas sociales de colectivos con necesidades particulares como son las familias extensas de predominio multigeneracional en las cuales suelen encontrarse las abuelas cuidadoras.

Por último, a partir de lo planteado en este artículo de reflexión teórica, se propone trabajar con la familias apoyándolas en la atención de sus necesidades, la resolución de sus problemas y en su función cuidadora, pero particularmente en el caso de las abuelas cuidadoras apoyándolas en sus actividades, ya que ellas pueden ser los miembros más sobrecargados. Una de las primeras formas para trabajar es hacer campañas de sensibilización comunitaria, para la movilización social ante situaciones que puedan provocar aislamiento o discriminación, para así promover el respeto y la cooperación ciudadana. Por lo tanto, la sensibilización sería la primera fase de un proceso de trabajo que se concretaría posteriormente en proyectos de intervención y actividades dirigidas a reducir los impedimentos culturales y sociales que limitan el acceso a sistemas naturales de apoyo más amplios y a los sistemas formales.

\section{CONCLUSIONES}

La familia ha sido considerada como una fuente básica de apoyo emocional en todas las etapas de la vida. En las familias extendidas en las que generalmente conviven tres generaciones (abuelos, padre o madre y nietos), la dinámica familiar puede caracterizarse por la ayuda y el apoyo emocional ante problemas económicos y familiares. Así, en situaciones de paternidad o maternidad adolescente, divorcio, ser madre soltera o cuando falta el cónyuge y también en situaciones de desplazamiento forzado, la familia extendida se convierte en una estrategia para sobrevivir y lograr cierto nivel de bienestar físico y psicológico.

Principalmente, las familias con miembros que se encuentran en la adultez tardía presentan ciertas características particulares, como la presencia de abuelos y la tendencia a que las familias sean multigeneracionales (incluyendo 3 ó 4 generaciones) (Ortiz y Carrillo, 2007). Así, en la relación entre abuelos, padres y nietos se establecen y fortalecen vínculos afectivos y de apoyo.

Los abuelos y las abuelas en su rol pueden experimentar muchas emociones y encontrar un mayor sentido de vida en la etapa del desarrollo en la que se encuentran. En nuestro contexto las familias valoran el rol de cuidadora de la abuela como parte fundamental de la red de apoyo familiar, pero el cuidado que ésta brinda puede implicar una sobrecarga en sus actividades y generar estrés, lo cual hace evidente la necesidad de intervención en las abuelas como una población en aumento que se encuentra en posible riesgo psicosocial.

Finalmente, se considera necesario incentivar la creación y fortalecimiento de programas que apoyen a las abuelas cuidadoras y a otros cuidadores familiares, para lo cual se requiere: proporcionar a las abuelas cuidadoras los conocimientos necesarios para que ellas tengan mayor seguridad en el cuidado que brindan, darles estrategias y técnicas para reducir la 
sobrecarga y el estrés, valorar sus sistemas de apoyo, impulsar las asociaciones de abuelas cuidadoras y de otros cuidadores familiares y, además, promover en los varones de la familia actitudes de apoyo social.

La psicología social comunitaria ha de tener en el futuro una visión más amplia de la función de las abuelas cuidadoras acorde a las condiciones sociales actuales y a los cambios en los sistemas familiares. Las investigaciones y las propuestas de intervención han de tener en cuenta factores individuales y de contexto (familiar, comunitario y macrosocial), dando mayor atención a los sistemas de apoyo implicados en los cuidados brindados por las abuelas y a las abuelas mismas.

\section{REFERENCIAS BIBLIOGRÁFICAS}

AINSWORTH, M. S. (1989). Attachments beyond infancy. American Psychologist, 44, $709-716$.

BARBER, C. E. (2001). Grandparents: Styles and satisfactions. Recuperado el 21 de Marzo de 2003, de http://www.ext.colostate.edu/pubs/consumer/10239.html

BENLLOCH, V., LLOPIS, D., BEJARANO, E. \& PINAZO, S. (1996). Estudio sobre la autopercepción de los abuelos en la relación con sus nietos. Información Psicológica, 61, $69-72$.

BELSKY, J.K.(1996). Psicología del envejecimiento: Teoría, investigaciones e intervenciones. Barcelona, España: Masson S.A.

BERK, L. (1999). Desarrollo del Niño y del Adolescente. 4ª Edición. Madrid: Prentice Hall.

BOWLBY, J. (1995). Vínculos afectivos: Formación, desarrollo y pérdida. 2a Edición. Madrid, España: Ediciones Morata.

BRONFENBRENNER, U. \& CECI, S. J. (1994). Nature-nurture reconceptualized in developmental perspective: A bioecological model. Psychological Review, 101, 568-586.

BROOKS-GUNN, J. \& CHASE-LANSDALE, P. L. (1995). Adolescent parenthood. En M. H. Bornstein, (Ed.), Hadbook of Parenting, 3, 113-149. New Jersey: LEA.

BUDINI, F. \& MUSSATTI, T. (1998). Grandmothers' involvement in grandchildren's care: Attitudes, feelings, and emotions. Family Relations, 48, 35- 42.

CANTOR, N. \& SANDERSON, C.A. (1999). Life task participation and well-being: The importance of taking part in daily life. Well-Being: the foundations of hedonic psychology, New York: Russell sage foundation. 
CARrillo, S., MALDONADO, SALDARRIAGA,L., VEGA, L. \& DÍAZ, S. (2004). Patrones de apego en familias de tres generaciones Abuela-madre adolescente-hijo. Revista Latinoamericana de Psicología, 36, 409-430.

CRAIG, G. J. (2001). Desarrollo psicológico ( $8^{\mathrm{a}}$ ed.). México: Prentice Hall.

COOPER, C. R. \& DENNER, J. (1998).Theories linking culture and psychology: universal and community specific processes. Annual Review Psychology, 49, 559-584.

DEPARTAMENTOADMINISTRATIVO NACIONAL DE ESTADÍSTICA(2005). Censo General Colombiano 2005. Recuperado el 12 de Febrero de 2007, de http://www.dane.gov.co.

DEPARTAMENTO ADMINISTRATIVO NACIONAL DE ESTADÍSTICA (2003). Encuesta Nacional de Calidad de Vida . Recuperado el 12 de Febrero de 2007, de http://www.dane.gov.co

DEPARTAMENTO ADMINISTRATIVO NACIONAL DE ESTADÍSTICA(2000). Especial: Envejecimiento de la población colombiana, desafíos inminentes. Boletín de Estadística. Santa fe de Bogotá, Colombia.

DEPARTAMENTO ADMINISTRATIVO NACIONAL DE ESTADÍSTICA(1998). La Familia Colombiana en el Fin de Siglo: Estudios Censales N ${ }^{\circ}$ 10. Santa fe de Bogotá, Colombia.

DULCEY-RUÍZ, E. (2004). La Situación de las Personas Mayores de 60 años en Colombia y las Políticas de Atención. Recuperado el 26 de Febrero de 2007, de http://www.sescsp. org.br/sesc/images/upload/conferencias/187.rtf.

DULCEY-RUÍZ, E. \& URIBE, C. (2002). Psicología del ciclo vital: Hacia un visión comprehensiva de la vida humana. Revista Latinoamericana de Psicología, 34, 17-27.

GAMBOA, J.J. (2007). Madres antes de los quince y abuelas cerca de los treinta: actitudes lingüísticas y culturales de las madres adolescentes en el desplazamiento forzado en Colombia. Göteborgs Universitet, Facultad de Ciencias Sociales. Recuperado el 20 de Febrero de 2009 de: http:/gupea.ub.gu.se/dspace/bitstream/2077/9977/1/haina_6_ gamboa.pdf.

GAMBOA, N \& CORDERO, A. (1998). Las redes de ayuda mútua: mecanismos de sobrevivencia. Revista de Ciencias Sociales, 44, 69-80.

GRANZIN, K. L. \& HAGGARD, L. M. (2000). Advances in Quality of Life Theory and Research: Social Indicator Research Series. Kluwer Academic Publishers.

GOMEZ, V. (2003). Riesgos de salud y su relación con el desempeño de múltiples roles en hombres empleados. Documentos CESO No 47, Universidad de los Andes. 
HARWOOD, R. L. (2002). Culture and Attachment: Perceptions of the Child in Context. New York: The Guildford Press.

KORNHABER, A. (1996). Contemporary Grandparenting. Sage Publications.

LIBERALESSO, A. (2002). Bienestar subjetivo en la vida adulta y en la vejez: hacia una psicología positiva en América Latina. Revista Latinoamericana de Psicología, 34, 55-74.

MARTÍNEZ, M. F. Y GARCA, M. (1995). La perspectiva psicosocial en la conceptualización del apoyo social. Revista de Psicología Social, 10, 61-74.

MENESES, R., FELDMAN, L. Y CHACON, G. (1999). Estrés, apoyo social y salud de la mujer con roles múltiples. Revista interamericana de Psicología, 33, 109-132.

MYERS, D. G. (1999). Well-Being: the foundations of hedonic psychology, New York: Russell sage foundation.

OCHAITA, E. \& ESPINOZA, M. A. (1995). Nuevas relaciones en el seno de las familias de finales del siglo XX: las relaciones entre miembros de generaciones alternas. Infancia y Sociedad: Revista de Estudios, 29, 27-46.

ORTIZ, M. J. \& CARRILLO, S. (2007). Influencia de Factores Sociodemográficos, Individuales y Relacionales en la Calidad de Vida y el Estrés de las Abuelas Cuidadoras, Bogotá: Universidad de Los Andes.

PAPALIA, D. E. \& WENDKOS, S. (1992). Desarrollo Humano (4ª ed.). Bogotá, Colombia: MacGraw Hill.

ROSENTHAL, C. J.\& GLADSTONE, J.(2000)Contemporary family trends: grandparenthood in Canada. The Vanier Institute of the Family. Recuperado el 16 de Abril de 2003, de http://www.vifamily.ca/cft/grandpt.

RUBIO, R. \& GARRIDO, J. A. (1995). Actividad social y redes sociales en relaciones intergeneracionales abuelos/as - nietos/as. Infancia y Sociedad: Revista de Estudios, 29, 165-184.

SANTANA, I. (2003). El apoyo formal a la familia como cuidadora natural. Portularia, 3, 117-135.

VILLALBA, C. (2002a). Abuelas cuidadoras: una Aportación para el Trabajo Social. Valencia: Tirant Lo Blanch.

VILLALBA, C. (2002b), Redes de apoyo social de abuelas cuidadoras. Portularia. Revista de Trabajo Social, 2, 263-282. 
REVISTA DE INVESTIGACIONES UNAD

WESTHEIMER, R. \& KAPLAN, S. (2000). Grandparenthood. New York: Routledge.

ZIRKEL, S. Y CANTOR, N. (1990). Personal construal of life tasks those who struggle for independence. Journal of Personality and Social Psychology, 58, 172-185. 